\title{
Star/Disk Interaction in the Nuclei of Active Galaxies
}

\author{
D. N.C. Lin
}

\section{Lick Observatory, University of California, Santa Cruz, CA 95064, US.A}

The most definitive evidence for an accretion disk is provided by the discovery of megamasers (Claussen et al. 1984) which appear to be located in a molecular torus around the nucleus of the mildly active galaxy NGC 4258. Confirmation of Keplerian rotation speed was obtained from radio interferometer (Nakai et al. 1993) and VLBI observations (Greenhill, this volume). Based on the correlation between the spatial locations and radial velocities of the masers, Moran et al. (1995) deduced that the masers are located in a disk at radii $R=0.13-0.26 \mathrm{pc}$ around a black hole with a mass $M \approx 3.5 \times 10^{\top} M_{\odot}$ (Watson \& Wallin 1994, Maoz 1995). A limit on the thickness $\left(H<2.5 \times 10^{-3} R\right)$ is deduced from the velocity dispersion of the maser sources. The mass-diffusion time scale is estimated to be $\tau_{d}>10^{15} \alpha^{-1} \mathrm{~s}$, where $\alpha$ is the viscosity parameter. An efficient angular-momentum transfer mechanism $(\alpha>0.1)$ is needed for $\tau_{d} \approx 10^{8} \mathrm{yr}$, which is the disk evolution time scale inferred from the correlation between interacting galaxies and intense AGN activities (MacKenty 1989, Hernquist 1989). A relatively large value of $\alpha$ is also required to account for the accretion rate needed to power the X-ray flux of NGC 4258.

Although MHD turbulence may be excited by magneto-rotational instabilities (Balbus \& Hawley 1991), its efficiency of angular-momentum transfer appears to be low $\left(\alpha<10^{-2}\right.$, Stone et al. 1996, Brandenburg et al. 1996). We suggest that star-disk interaction may lead to $\alpha \approx 1$. In the nucleus of $M 87$, a cusp in the stellar density extends into a rotating gaseous disk (Lauer et al. 1992). The passage of most field stars through the disk excites corrugated waves at their vertical resonances (Artymowicz 1993). The dissipation of these waves induces the deposition of the stellar kinetic energy into the disk and a gradual decrease in the stars' orbital inclination (Syer et al. 1991). Stars with relatively low initial inclination essentially corotate with the disk and are rapidly trapped by the disk. In the dense environment of the disk, the trapped stars gain mass as they accrete gas. Despite their tidal interaction with the disk gas, stars trapped at $R>10^{-2}$ pc are likely to evolve into type II supernovae (Artymowicz et al. 1993). The supernova explosions introduce a local stirring which leads to an efficient outward transfer of angular momentum (Rozyczka et al. 1995). The remnants also contaminate the disk with heavy-element ejecta. The concurrent contamination by trapped stars and the flushing of disk gas into the central massive black hole induce the disk metallicity to be comparable to or larger than that of the Sun. This model naturally accounts for the super-solar heavyelement abundance of quasars (some with large redshifts) which is inferred from the observed emission-line intensities of the Fe and the CNO group of elements (Davidson 1977; Rees et al. 1989; Hamann, this volume).

The distribution of Galactic pulsars indicates that typical neutron-star remnants have typical recoil velocities $\sim 100 \mathrm{~km} \mathrm{~s}^{-1}$ (Narayan \& Ostriker 1990, Lyne 
\& Lorimer 1994), which is much smaller than the Keplerian speed in the disk of NGC 4258. Thus, the supernova remnants are likely to be retrapped with small orbital inclination. Upon settling back into the disk, the compact remnants resume their rapid accretion despite the Eddington-limit barrier (Chevalier 1993) and become stellar-mass black holes ( $\mathrm{SBH}$ ) on time scales short compared with $\tau_{d}$. When these SBH have acquired a mass $\sim(H / R)^{3} M \approx 10^{2} M_{\odot}$, they are able to open gaps in the vicinity of their orbit (Lin \& Papaloizou 1986) and the quench their growth. The SBHs also exchange orbital angular momentum with the disk gas near their orbital radius through tidally induced density waves. Embedded in the background of many low-mass field stars, the orbits of the SBHs decay by dynamical friction. The excess angular momentum received from the disk is then passed to the field stars in the galactic nucleus, which in turn undergoes two-body relaxation with more distant stars. A self-consistent estimate indicates that at any given time, $\sim 10^{4} \mathrm{SBH}$ may be embedded in the disk and their collective contribution would drive the disk to evolve on a time scale $\sim 10^{8} \mathrm{yr}$ with an corresponding $\alpha \approx 1$ (Lin et al. 1994).

Acknowledgments. This work is supported by NASA grant NAG5-3059.

\section{References}

Artymowicz, P. 1994, ApJ, 423, 581.

Artymowicz, P., Lin, D. N. C., \& Wampler, E. J. 1993, ApJ, 409, 592.

Balbus, S. A., \& Hawley, J. F. 1991, ApJ, 376, 214.

Brandenburg, A., Nordlund, A., Stein, R.F., \& Torkelsson, U. 1996, ApJ, 458, L45.

Claussen, M.J., Heiligman, G. M., Lo, K.Y. 1984, Nature, 310, 298.

Chevalier, R. 1993, ApJ, 411, L33.

Davidson, K. 1977, ApJ, 218, 20.

Hernquist, L. 1989, Nature, 340, 687.

Lauer, T. R, et. al. 1992, AJ, 103, 703.

Lin, D. N.C., Artymowicz, P., \& Wampler E. J. 1994, in Theory of Accretion Disks-2, eds. W.J. Duschl et al. (Dordrecht: Kluwer), 235.

Lin, D. N. C., \& Papaloizou, J. C. B. 1986, ApJ, 307, 395.

Lyne, A. G., \& Lorimer, D. R. 1994, Nature, 369, 127.

Mackenty, J.W. 1989, ApJ, 343, 125.

Maoz, E. 1995, ApJ, 447, L91.

Moran, J., et al. 1995, Proc. Nat. Acad. Sci., in press.

Nakai, N., Inoue, M., \& Miyoshi, M. 1993, Nature, 361, 45.

Narayan, R., \& Ostriker, J.P. 1990, ApJ, 352, 222.

Rees, M. J., Netzer, H., \& Ferland, G. J. 1989, ApJ, 347, 640.

Rozyczka, M., Bodenheimer, P. H., \& Lin, D. N.C. 1996, MNRAS, 276, 597.

Stone, J. M., Hawley, J. F., Gammie, C. F., \& Balbus, S. A. 1996, ApJ, 463, 656.

Syer, D., Clarke, C. J., \& Rees, M. J. 1991, MNRAS, 250, 505.

Watson, W. D., \& Wallin, B. K. 1994, ApJ, 432, L35. 